\title{
Mapas cognitivos: Revisión crítica
}

\section{Manuel Carreiras}

Universidad de La Laguna

Muchos aspectos de nuestra conducta en el ambiente indican que poseemos una representación interna del mismo. Somos capaces de planificar la ruta hasta un lugar determinado y llegar alli sin demasiados problemas. Incluso, si una calle está cortada buscamos con facilidad una senda alternativa. Con frecuencia podemos dar las instrucciones precisas a un forastero para que se encamine en la dirección correcta y alcance el lugar deseado; rara vez nos perdemos en la ciudad en que vivimos, etc. La realización de éstas y otras tareas similares requieren, en ausencia de ayudas externas, tales como un mapa, la presencia de una representación interna - mapa cognitivo- que preserve información sobre el ambiente.

En las últimas décadas se ha dedicado una cantidad considerable de esfuerzos al estudio del conocimiento ambiental. Atrajo la atención de investigadores procedentes de diversas disciplinas: geógrafos, psicólogos, arquitectos, etc., que le han conferido a esta reciente área de estudio un carácter multidisciplinar (Canter y Craik, 1981; Craik, 1973, 1977; Garling et al. 1984a; Jimenez Burillo, 1981; Stokols, 1978). Así pues, el tema se ha abordado desde diversos puntos de vista. Los geógrafos han dirigido más su foco de atención hacia los aspectos simbólicos y evaluativos que modulan la representación (Lowenthal y Riel, 1972; Gould, 1974), aunque también se han ocupado de cómo construimos la representación interna del ambiente (Capel, 1973; Golledge, 1978). Los arquitectos se han interesado más por cuestiones concernientes al diseño (Weisman, 1981; Kuller, 1977a, 1977b) así como por el influjo de la estructura urbana y las características físicas de los edificios en la imagen de la ciudad (Lynch, 1960; Appleyard, 1970; Zannaras, 1976). La Psicología ha estudiado el área desde una cuádruple vertiente. Los psicólogos ambientales han investigado principalmente cómo se percibe el am- 
biente y cuáles son las dimensiones o factores que subyacen al significado que le conferimos (Horayangkura, 1978; Ward y Russell, 1981a y 1981b). Los psicólogos evolutivos han intentado explicar cómo las representaciones y las destrezas manipulativas cambian con el desarrollo (Siegel et al. 1978; Spencer y Weetman, 1981; Pick y Rieser, 1982). Los psicólogos del procesamiento de información han situado el énfasis en el formato de la representación interna, así como en los procesos de adquisición, orientación y planificación de la conducta (Stevens y Coupe, 1978; Goldin y Thorndyke, 1982; Lindberg y Garling, 1982, 1983; Garling et al. 1982, 1983). Por último, cabe resaltar también el acercamiento psicofisiológico (O'Keefe y Nadel, 1978; Lieblich y Arbib 1982).

Esta riqueza de perspectivas condujo a la acumulación de una ingente cantidad de datos, que a su vez va unida a una cierta debilidad teórica. Se ha observado que la precisión localizacional se incrementa con la edad (p.e. Allen et al. 1979), con la familiaridad (p.e. Garling et al. 1981); que las características físicas del medio, como los mojones, barreras, cambios de dirección e incluso el color interno de los edificios (Evans, 1980) afectan a la representación interna del espacio. También se ha comprobado que las demandas atencionales dependen de la naturaleza de los lugares (Lindberg, 1984), que la representación puede adquirirse de forma distinta a través de sentencias, estudiando mapas cartográficos o por deambulación (Foos, 1980; Thorndyke y Stazs, 1980), etc. Sin embargo, por el momento no existe un marco conceptual satisfactorio, que integre esta multitud de hallazgos, aunque las revisiones de Garling et al. (1984a) y de Evans (1980) han supuesto un gran avance en esta dirección.

A lo largo de esta revisión intentamos ofrecer un análisis de la noción de mapa cognitivo desde la perspectiva del procesamiento de información, con el fin de sugerir un marco conceptual donde integrar y contrastar algunos resultados notables de la literatura experimental. Diversas cuestiones se han omitido conscientemente o han sido abordadas superficialmente, como por ejemplo los procesos de adquisición del mapa. Pero la descripción detallada de todas las líneas de investigación excede el objetivo de estas páginas. Además existen buenas revisiones que abordan otros aspectos del mapa cognitivo (Garling et al. 1984a; Evans, 1980; Pick y Rieser, 1982; Russell y Ward, 1982).

La premisa básica, en torno a la que se construye nuestra revisión, consiste en la consideración conjunta de factores espaciales y semánticos. Intentamos conjugar los dos componentes del mapa cognitivo, localizacional y atributivo-contextual, con frecuencia aislados por motivo de análisis, y apresar su interacción en la explicación de las distorsiones e inconsistencias que sufre la representación interna del ambiente. 


\section{CONOCIMIENTO ESPACIAL}

El conocimiento de espacios de gran escala puede provenir de muchas fuentes de información. Un plano de la ciudad o del metro proporciona un simbolismo conciso para una gran cantidad de información geográfica. Pero, con frecuencia, uno no aprende el trazado de la ciudad donde vive memorizando un mapa cartográfico, sino a partir de la experiencia de desplazarse por ella. Se observan edificios, plazas, rutas que los unen, se codifica esta información y se va integrando en una representación coherente. En este sentido, el conocimiento del ambiente no sólo implica un registro visual de los elementos del medio, sino que también se refiere a sucesos, similitudes conceptuales, sentimientos y significado personal que conferimos a los diferentes lugares. No adquirimos una copia fiel de la realidad; cada persona ve y reconstruye el mundo a través de sus propios supuestos, valores y experiencias vitales. La representación interna de este cúmulo de información ha recibido diversas denominaciones. Las tres más utilizadas han sido: imagen, esquema y mapa cognitivo. Estos conceptos intentan apresar y definir el contenido y procesos subyacentes a la representación, aunque han visto la luz en distintos contextos y cada uno enfatiza aspectos diferentes.

El significado psicológico de la palabra imagen fue delimitado por Boulding (1956), quien sugiere que los individuos desarrollan, a lo largo de su vida, impresiones mentales del mundo a través de contactos diarios con el ambiente. Estas imágenes y no el ambiente físico son los determinantes de la conducta. La creación de la imagen ambiental es un proceso de interacción entre el observador y lo observado. Apresamos la realidad externa, pero la forma en que la interpretamos influye en lo que extraemos (Lynch, 1960). Así pues, la estructura básica de la imagen es un extracto simplificado de la realidad, mediatizado por procesos individuales.

La característica fundamental de las imágenes es su carácter analógico. Guardan un cierto isomorfismo con la realidad, pero no son copias fieles y exactas de la misma. La teoría más aceptada hoy día (Kosslyn y Shwartz, 1977; de Vega, 1979; Kosslyn, 1980) postula que son representaciones espaciales que ocurren en la memoria activa, generadas y no simplemente recuperadas de representaciones más abstractas, almacenadas en la memoria a largo plazo. Las imágenes se construyen usando tanto información perceptiva como conceptual, que ha sido previamente codificada, y sufren las limitaciones de la memoria a corto plazo. Así, por ejemplo, tienen una resolución muy baja, especialmente en los bordes y una estructura temporal, de modo que su activación es secuencial y sus partes se deterioran con rapidez.

Los esquemas se han definido como estructuras básicas dentro de las que se organizan las experiencias presentes y pasadas. El supuesto básico que subyace a este concepto es que la experiencia an- 


\section{Estudios}

terior del individuo influye en como percibe, comprende y recuerda información nueva (Rumelhart, 1980). Se entiende por esquemas aquellas estructuras cognitivas o sistemas de codificación que permiten al individuo responder a un patrón de estímulos ambientales en continuo cambio. Son dinámicos y a la vez selectivos en lo que incorporan (Neisser, 1976).

La noción de esquema proviene del trabajo de Bartlett (1932) sobre percepción de objetos, rostros, comprensión de historias y fue trasladada a contextos ambientales por Lee (1968). Posteriormente, ha sido adoptada por una gran cantidad de investigadores como un concepto descriptivo o explicativo de la estructura y organización del conocimiento, que dirige o guía la atención, interpretaciones, inferencias y expectativas del individuo (Bobrow y Norman, 1975; Schank y Abelson, 1977; Rumelhart y Ortony, 1977; Kintsch y Dijk, 1978). El término se ha aplicado en un vasto dominio. Entre los numerosos ejemplos de la literatura psicológica que sugieren una interpretación posible mediante la teoría de los esquemas se encuentra la percepción y el recuerdo de objetos y lugares (Minsky, 1975; Neisser, 1976). La percepción espacial es un proceso constructivo que implica entre otras cosas la extracción de características (procesos de abajo-arriba), pero también la confirmación de expectativas y la interpretación en base al conocimiento previo, esquemas y metas (procesos de arriba-abajo). Salmaso et al. (1983) y Brewer y Treyens (1981) encontraron que se recuerda mejor la información sobre lugares congruente con un esquema previo sobre los mismos, que aquella no relevante o inesperada según el esquema. El efecto de la congruencia, sin embargo, está modulado por la saliencia de la información así como por la adopción de estrategias intencionales o incidentales y no favorece el reconocimiento.

Esquema, estructura, script y otros conceptos similares en general han estado vinculados a los modelos de representación proposicional, excepto en algunos modelos, entre los que cabe citar el de Neisser (1976), el de Minsky (1975), o la formulación proposicional un tanto peculiar de Schank y Abelson (1977). Estos permiten explicar mediante un formato de representación abstracto fenómenos de percepción, comprensión, recuperación, etc. y mimetizar las explicaciones y predicciones de las teorías de las imágenes (Anderson, 1978; Pylyshyn, 1981).

El término mapa cognitivo fue acuñado por Tolman (1948) con ánimo de definir el carácter espacial de la imagen ambiental, en oposición a las teorías conexionistas de su época. La interpretación literal del término ha conducido a enfatizar que sus propiedades guardan una cierta analogía con las de un mapa cartográfico. Esta concepción ha recibido muchas críticas, ya que induce a considerarlo únicamente como una representación estática y euclidiana de carácter analógico (Downs, 1981; Kuipers, 1982). Pero aunque el término mapa cognitivo hace referencia a una representación interna, algu- 
na de cuyas funciones se corresponde con las de un mapa cartográfico, no significa que adopte tales propiedades físicas (Blaut, McClearly y Blaut, 1970). Los mapas cartográficos son modelos de la realidad a escala. En cambio, los mapas cognitivos son incompletos, esquemáticos, algunas de sus partes se ven aumentadas y otras disminuidas con respecto a la realidad que distorsionan y simplifican; poseen además un componente dinámico y ejecutivo dirigido a la acción.

Categorizamos, clasificamos e imponemos una estructura a la representación ambiental a fin de convertirla en algo manejable, predecible y mínimamente ordenado. Esta estructura cognitiva, llamada también esquema perceptivo, prepara al observador para áceptar cierto tipo de información y dirige su exploración (Neisser, 1976). Por otra parte, el mapa cognitivo no se reduce a un conocimiento de la disposición relativa de lugares y rutas; incluye también información conceptual sobre el medio y además un conocimiento procedimental acerca de su utilización. Constituye, en suma, un sistema de razonamiento espacial, que nos permite planificar conductas y resolver problemas ambientales (Downs y Stea, 1977). Es una herramienta básica para la adaptación al ambiente, ya que se muestra necesario tanto para la supervivencia humana (Kaplan, 1976; Kearins, 1981), como para la conducta espacial cotidiana (Garling et al., 1982, 1984a).

De acuerdo con esta argumentación, las líneas divisorias entre imagen, esquema perceptivo y mapa cognitivo se difuminan un poco. No abrigamos la pretensión de suponer un total solapamiento entre los tres términos. Mas bien hemos relatado el hecho de que existen algunas semejanzas conceptuales, que nos inducen a considerar, junto con una base empírica de datos, el mapa cognitivo bajo una perspectiva representacional múltiple: analógica, conceptual y procedimental (cfr."de Vega, 1982; Carreiras y de Vega, 1984).

\section{ELEMENTOS DEL MAPA COGNITIVO}

El ambiente es complejo y rico en información. El área física que percibimos en un momento dado es pequeña, está restringida por el alcance de nuestros sentidos y por las barreras que nos circundan. Aunque podemos percibir pocas cosas a la vez, compensamos esa restricción de varias formas. La capacidad de movimiento nos permite explorar más áreas y ampliar el contacto con el medio. La visibilidad de algunos edificios desde lugares diferentes nos proporciona anclajes de orientación y el esqueleto para la organización de uno o varios sistemas de referencia. Sin embargo, el muestreo experiencial que realizamos es sesgado y no sistemático, aunque nuestros patrones de desplazamiento no son aleatorios, sino que tienden a seguir ciertos trayectos (vg: al trabajo, al cine, etc.). 


\section{Estudios}

Así pues, estructuramos nuestro conocimiento a partir de un conjunto informacional discreto, extraído de diversas partes del ambiente.

Aunque en la experiencia ambiental se incorpora información a través de todos los sentidos, el predominio es visual. Desde esta perspectiva, Lynch (1960) construyó una tipología de elementos para describir la estructura cognitiva de la imagen de la ciudad. Basándose en los dibujos de mapas de tres ciudades, realizados por sus habitantes, identificó cinco componentes de la imagen urbana. Sendas o rutas por las que nos movemos para ir de un lugar a otro. Distritos o barrios de la ciudad, caracterizados por alguna propiedad geográfica o cultural reconocida. Limites de regiones, áreas o barrios. Nodos, puntos salientes donde confluyen varios trayectos. Mojones o hitos, que se refieren a lugares o edificios perceptivamente salientes o distintivos, fácilmente reconocibles en la lejanía. Esta ciasificación ha recibido apoyo empírico (Magaña, 1978; Aragonés, 1983). Sin embargo, Siegel y White (1975), Kuipers (1978), Thorndyke (1981), Hernández (1984) han apuntado que de los cinco elementos son las sendas y mojones los más relevantes, y posiblementes suficientes para poder operar en el ambiente. No obstante, además admiten que las representaciones más sofisticadas implican un conocimiento de las relaciones espaciales de localización, al que atribuyen cualidades gestálticas. Este tipo de representación configuracional permite relacionar lugares, facilitando las inferencias a partir de la información de sendas o trayectos; o bien cómputos más abstractos como la estimación de distancia en línea recta entre lugares y las estimaciones angulares de dirección.

Los tres elementos de esta clasificación más parsimoniosa - mojones, sendas y relaciones espaciales- pueden conceptualizarse como diferentes tipos de conocimiento o etapas progresivas de representación. Varios autores (Appleyard, 1970; Shemyakin, 1962; Siegel, Kirasic y Kail, 1978; etc.), utilizando a veces terminologías diferentes, han argumentado que el conocimiento ambiental progresa desde la representación de puntos de referencia y sendas que los conectan hasta una visión configuracional.

\subsection{Puntos de referencia}

En el proceso de aprendizaje de un ambiente nuevo establecemos, en primer lugar, el esqueleto de la representación interna, en base a una serie de lugares como el de residencia, trabajo y sitios de recreo frecuentados, etc. A medida que nuestro espacio de actividad se expande exploramos más lugares, que representamos en relación a los que previamente conocíamos. Organizamos el mapa cognitivo según una jerarquía funcional de lugares, donde algunos cobran más importancia en función del significado que les conferimos, la familiaridad u otros criterios de naturaleza perceptiva (Carr 
y Schissler, 1969; Golledge, 1978). Los lugares de referencia son representados con mayor precisión y detalle que los restantes, y desempeñan un papel especial en los procesos de orientación. Conocemos relativamente bien su localización espacial, convirtiéndose así en puntos de anclaje, que sirven para definir el emplazamiento de otros lugares adyacentes o cercanos (Sadalla et al., 1980; Lindberg, 1984). Por ejemplo, en orden a determinar en la ciudad de Madrid la localización de una tienda cercana a la «Plaza de Colón», es plausible suponer que recuperamos primero el emplazamiento de la «Plaza de Colón», luego, en relación a ésta, inferimos el de la tienda. Cuando alguien nos pregunta dónde se halla situado un determinado lugar, solemos orientarlo con respecto a puntos de referencia. Con frecuencia decimos la tienda «X» está junto a la «Plaza de Colón», pero nunca, a menos que la tienda sea un lugar prominente, que la «Plaza de Colón» se encuentra al lado de la tienda «X».

De acuerdo con la terminología de Lynch, un punto de referencia podría ser tanto un mojón como un nodo, pero no existe una correspondencia conceptual exacta. Dependiendo de la escala espacial en que nos movamos, un distrito o una ciudad entera pueden considerarse también puntos de referencia. Potencialmente, cualquier elemento ambiental puede servir como punto de referencia, sin embargo, sólo algunos son considerados como tal, debido a su elevada representatividad o porque elicitan un significado distintivo.

El predominio de ciertos detalles de los lugares proviene a menudo de nuestro interés particular o actitudes, debido a una serie de episodios personales con los que lo hemos ligado; pero puede resultar también de un determinado significado convencional o simbólico, o de algunas características estructurales del lugar (Lieblich y Arbib 1982). Appleyard (1969) aisló una serie de atributos que contribuyen a que algunos lugares capten más nuestra atención que otros. Los criterios determinantes del mejor recuerdo e identificación de los edificios fueron: Las características distintivas de la forma física, como tamaño, configuración, aspecto externo; características perceptivas de saliencia y visibilidad; funcionalidad de uso e importancia cultural. "Los índices de Appleyard fueron corroborados por Evans, Smith y Pezdek (1982), quienes incorporan tres adicionales: forma artística, estilo arquitectónico y estructura diferenciada. Retomando la investigación de Appleyard, Sadalla et al. (1980) construyeron 18 escalas bipolares, cuyas puntuaciones sobre una serie de lugares sometieron a regresión múltiple. La variable dependiente era un índice de referencialidad de los lugares, extraído de acuerdo con la metodología de Rosch (1975). El análisis de regresión indicó que las escalas de familiaridad, visible a distancia, dominancia de los lugares cercano e importancia cultural, predecían significativamente las puntuaciones del índice de referencialidad. 


\subsection{Sendas}

Las sendas son unidades representacionales importantes en el proceso de adquisición del mapa cognitivo en cuanto que sirven para expandir e integrar el conocimiento espacial, conectando experiencias perceptivas de lugares distantes. Una senda puede concebirse como una secuencia ordenada de lugares. Pero, además de la información serial, el conocimiento de la senda implica asociar a cada lugar la conducta pertinente para poder continuar el viaje a través de la ruta, y llegar al próximo lugar de la secuencia. (Por ejemplo, lugar A-girar a la derecha, lugar B-girar a la izquierda, lugar C-seguir de frente). Estas asociaciones se asemejan según Thorndyke (1981) a reglas de producción del tipo «si mi destino es «X» y estoy en «Y», entonces ejecutar acción «Z», o a lo que Clayton y Woodyard (1981) denominan grupos de «situación-acción-resultado». La ordenación de eslabones situación-acción o reglas de producción, constituye el conocimiento procedimental de sendas y nos permite alcanzar el destino al que nos dirigimos. Así pues, en la representación de sendas o rutas incorporamos una secuencia temporal de imágenes sensoriales y operaciones motoras mediante las cuales registramos la distancia recorrida y los giros entre segmentos de la ruta (Kuipers, 1978). Excepto en el caso infrecuente de que la senda sea completamente recta, podemos dividirla en segmentos separados por cambios de dirección. La evidencia empírica nos invita a pensar que tendemos a codificar los cambios de dirección como ángulos rectos (Byrne, 1979; Moar y Bower, 1983). Asimismo, se asume que en el mapa cognitivo representamos alguna información acerca de la longitud de los segmentos (Sadalla et al., 1979; Sadalla y Staplin, 1980a).

El concepto de senda así entendido evoca similitudes con el de «Planes de desplazamiento» (Garling et al., 1984a; Russell y Ward, 1982), derivado a su vez de la noción de planes jerárquicos como estructura organizativa de la conducta (Miller, Galanter y Pribram, 1960). Garling y colaboradores asumen que los planes de desplazamiento consisten en grupos ordenados de lugares, constituyendo así las sendas planes o parte de ellos. Por ejemplo, la senda desde casa al trabajo puede constituir en algunas ocasiones un plan más amplio, que incluye además ir del trabajo al puerto o a otro sitio. Kuipers (1978) define las sendas como un grupo de instrucciones que especifican cómo desplazarse. Los planes especifican, aunque sea parcialmente o de forma esquemática, cómo ir de un lugar a otro, requiriendo instrucciones más detalladas de cómo desplazarse durante su ejecución. Generalmente deambulamos por la ciudad con un plan preconcebido de alcanzar una meta. Así pues, plan de desplazamiento y senda pueden significar lo mismo en las etapas iniciales de la adquisición del mapa cognitivo, ya que no hemos establecido aún un intrincado grupo de relaciones espaciales abstractas 
entre lugares. En etapas más avanzadas de conocimiento del medio, algunas fases específicas del plan pueden implicar representación de sendas. Sin embargo, el esbozo general del plan sin duda se establece en base a una representación más abstracta de relaciones espaciales entre lugares. Además, la planificación de la conducta en ámbitos urbanos incluye reglas y metas con diferentes niveles de abstracción (cfr. Passini, 1977; Hayes-Roth, 1979; Garling et al. 1982).

\subsection{Relaciones espaciales}

A medida que se incrementa la familiaridad con el ambiente, se adquiere conocimiento de una serie de relaciones entre lugares, no directamente perceptibles, y de sus posiciones en el medio. Este tipo de información define el conocimiento configuracional o survey map (Shemyakin, 1962, Hart y Moore, 1973), o también llamado «mapa predominantemente espacial» (Appleyard, 1970).

$A$ veces se cae en la tentación de asumir que el formato representacional del conocimiento configuracional es similar al de un mapa cartográfico. Sin embargo, la literatura experimental ha demostrado que el mapa cognitivo no preserva con exactitud relaciones métrico-euclidianas entre los lugares. Por el contrario, es esquemático y distorsionado, lo que ha inducido a postular la coexistencia de al menos tres tipos de relaciones: de proximidad, métricas y de inclusión (Siegel y White, 1975; Kuipers, 1978; Garling et al., 1984a).

Stevens y Coupe (1978) proporcionaron evidencia congruente con la idea de relaciones de inclusión. Las relaciones espaciales entre ciudades son recordadas en función de las relaciones que mantienen entre sí los estados en las que están incluidas. Ello induce a errores cuando hay incongruencia entre las posiciones de las ciudades y sus respectivos estados (vg: la ciudad $a$ está al norte de la ciudad $b$, pese a que el estado $A$ que contiene a $a$ es meridional respecto al estado $B$ que contiene a $b$ ). Este principio de anidamiento puede suponerse también en otra escala espacial, como lugares de una ciudad. Por ejemplo, la tienda «X» puede recordarse incluida en el edificio «Y», el edificio «Y» dentro del distrito «Z», etc. En cuanto a las relaciones métricas y de proximidad, se ha asumido previamente que la representación de los lugares de referencia es detallada, mientras que la localización de otros menos importantes se infiere en relación a ellos. Es plausible suponer que entre los puntos de referencia se establecen relaciones espaciales métricas (relaciones geométricas euclidianas y no euclidianas) de dirección y distancia (Garling et al., 1984a; Golledge, 1978). En cambio, serían relaciones de proximidad las que definiesen la localización de otros lugares con respecto a los puntos de referencia (Acredolo et al., 1975; Sherman et al., 1980; Sadalla et al., 1980). 
El supuesto de que se codifiquen las relaciones métricas no implica necesariamente que se representen con absoluta precisión. La gran cantidad de información existente en el medio y las restricciones de nuestra sistema cognitivo hacen necesaria la utilización de reglas de simplificación o heurísticos, que introducen distorsiones (Tversky, 1981). Por otra parte, el uso de diferentes sistemas de referencia puede ser causa de inexactitudes. Kuipers (1978) argumenta que diferentes puntos de referencia podrían estar definidos respecto a diferentes sistemas de referencia locales, que difieren en orientación o incluso en escala. En este caso, la metáfora del mapa cognitivo, que supone una estructura unitaria de orientación global, podría modificarse transformándola en la de un atlas mental con múltiples sistemas de orientación (Russell y Ward, 1982). Un lugar podría estar representado con respecto a más de una sistema de referencia, para hacer posible, a través de métodos de resolución de problemas, deducciones de la relación entre dos estructuras o lugares pertenecientes a estructuras diferentes. De esta forma, se predice que las relaciones entre lugares dentro de una estructura de referencia son representadas con gran precisión (Siegel y Schadler, 1977). Por el contrario, existe poca precisión entre lugares pertenecientes a estructuras diferentes ya que las inferencias necesarias para obtener su relación introducen distorsiones sistemáticas. Otro corolario que se desprende de esta argumentación es que las relaciones espaciales métricas (dirección y distancia), desde un lugar a otros, están vinculadas a un sistema de referencia; pero las relaciones métricas inversas, es decir, desde los otros lugares al primero, se recuerdan con respecto a sistemas de referencia diferentes.

\section{CONTENIDO DEL MAPA COGNITIVO}

- Dos son los tipos de información que preserva el mapa cognitivo: localizacional y atributiva. La información localizacional nos indica dónde se encuentra un determinado lugar, mientras que el conocimiento de cómo son los lugares, qué función desempeñan, qué acontecimientos suelen ocurrir en ellos y cuándo ocurren, correspondería al componente atributivo. La suma de ambos tipos de información - atributiva y localizacional- en un mapa cognitivo, nos permite operar en el ambiente (Downs y Stea, 1973, 1977), mediante procesos ejecutivos consustanciales como toma de decisiones, planificación, etc.

\subsection{Información localizacional}

La información localizacional puede mostrarse bajo dos aspectos, como una descripción estática o dinámica. Se puede inferir dónde se encuentra un determinado lugar, recordando la conducta rea- 
lizada para llegar hasta él. Es decir, en función de los lugares de partida y meta y de las rutas que los conectan. Asimismo, podemos describir su posición con respecto a otros lugares, o atendiendo a una estructura de referencia. Estas dos vertientes del conocimiento localizacional se corresponden con la dicotomía entre representación secuencial o procedimental y representación configuracional (Siegel y White, 1975; Thorndyke 1981).

El conocimiento procedimental hace referencia a la representación de rutas entre lugares, consiste en un registro de indicios entre el punto de partida, mojones intermedios y el lugar de destino. Entre estos indicios se incluye la percepción de la distancia de los tramos de la ruta, así como los cambios específicos de dirección que hay que realizar para llegar a la meta.

El conocimiento configuracional supone un salto cualitativo. Implica la representación de la localización de los lugares con respecto a un sistema de referencia global o local (cfr. Garling et al., 1984b), así como de las relaciones de distancia. Este tipo de información es fácilmente apresable en un plano bidimensional, pero no ocurre lo mismo cuando nos encontramos inmersos en el ambiente, ya que nos rodea y no podemos verlo como un todo, en conjunto. En contacto con el ambiente adquirimos un conocimiento secuencial. Aprendemos lugares, secuencias de acciones para movernos entre ellos e impresiones de distancias de las rutas. Solo cuando la familiaridad con la ciudad se incrementa, la representación se va reorganizando hacia la consecución de un conocimiento configuracional.

La localización de un lugar, bien sea a través de una descripción dinámica o estática, se deriva del conocimiento de su orientación y de la separación espacial con respecto a nuestra posición o a la de otros lugares. El mapa cognitivo en su aspecto localizacional contiene información acerca de la dirección y distancia entre lugares.

\subsubsection{Distancia}

Estimar la distancia que existe entre dos lugares de la ciudad sin la ayuda de un plano es una de las habilidades funcionales que con frecuencia utilizamos. La investigación sobre distancia cognitiva ha intentado conocer el grado de precisión con que las estimaciones se ajustan a las distancias reales, aislar las variables que inciden en las distorsiones que se producen y, desarrollar modelos explicativos de la naturaleza de las distorsiones.

Una evaluación del progreso empírico y teórico del campo precisa de la clarificación previa de una serie de deficiencias metodológicas, que dificultan la comparación de resultados y el contraste de hipótesis. (Véase Canter, 1977 y Cadwallader, 1979 para una revisión). En algunos estudios se les pidió a los sujetos que comparasen las distancias dos a dos o con otra distancia patrón y expre- 
saran una en proporción a la otra -método de razón de distancias- (Golledge et al., 1969; Lowrey, 1970; Briggs, 1973). En otros se ha utilizado la estimación directa de la distancia física (Lee, 1970; Cadwallader, 1973), estimación en metros (Lindberg, 1981, 1982) y en millas (Canter y Tagg, 1975). Cadwallader (1979) demostró que la distancia estimada no es totalmente independiente de la metodología empleada. Así pues, la comparación de resultados debe acometerse con cautela. La diversidad de métodos puede explicar parte de la diversidad de resultados obtenidos por los diferentes estudios.

Otra fuente de posible confusión proviene de la escala de los estímulos empleados en los experimentos. Son diferentes los mecanismos cognitivos implicados en la estimación de distancias entre ciudades del mundo (Lundberg, 1973; Ekman y Bratfish, 1965) y entre lugares de una ciudad (Briggs, 1973; Lee, 1970) o de un edificio (Lindberg, 1981, 1982). El proceso de aprendizaje del mapa cognitivo de una ciudad es bastante diferente del de un mapamundi. En el primer caso la representación adquirida está mediatizada por la experiencia directa con el medio. No percibimos el trazado completo de la ciudad en conjunto, a menos que utilicemos un plano, ya que nos rodea, estamos inmersos en ella. Construimos la imagen de la ciudad a partir de la acumulación progresiva de datos separados en el espacio y el tiempo. Por el contrario, en un mapa podemos percibir la distancia en línea recta entre dos ciudades, así como su orientación con respecto a un sistema de referencia global y coordinado. Así pues, podemos aprender esas relaciones directamente y recuperarlas sin necesidad de recurrir a un proceso transformacional complejo (Thorndyke y Hayes-Roth, 1982).

Por último, incluso manteniendo constante la escala del medio empleado, las diversas tareas de estimación requieren acudir a representaciones del espacio cualitativamente diferentes o utilizar procesos inferenciales distintos. No es lo mismo estimar la distancia del trayecto o la ruta entre dos lugares (Sadalla y Magel, 1980; Byrne, 1979), que realizar la estimación en línea recta (Canter y Tagg, 1975; Lindberg y Garling, 1983). Si hemos adquirido la disposición espacial de los lugares mediante experiencia directa con el medio, no es preciso recurrir a una representación configuracional para realizar la estimación de distancia de una ruta. Las operaciones mentales necesarias son relativamente simples, como por ejemplo sumar la distancia de cada tramo, basándonos únicamente en un recorrido imaginario de los mismos. Por el contrario, para estimar la distancia en línea recta, necesitamos acudir a una representación de la relación espacial de los dos lugares, ya consolidada con la experiencia o adquirida de un mapa cartográfico; o bien, en las primeras etapas del aprendizaje directo, realizar operaciones mentales bastante complejas con la información que nos proporciona el recorrer mentalmente la ruta. Sin embargo, con el mapa cognitivo 
consolidado, la tarea de estimación directa es bastante más intuitiva y sin duda menos compleja que lå de juzgar la distancia de una ruta.

A pesar de la confusión metodológica apuntada, el patrón común de resultados sugiere que existe cierta correspondencia entre la distancia cognitiva y la real. La evidencia en cuanto a la naturaleza exacta de la relación es, sin embargo, contradictoria. Gollege y Zannaras (1973) y Cadwallader (1976) entre otros, obtuvieron una relación lineal, mientras que los datos de Brafisch (1969), Golledge et al. (1969) y Briggs (1973) se ajustan mejor a una función curvilínea. Esta tendencia no es constante, sino que la no-linearidad se acentúa con las distancias más largas. Como ya se ha sugerido en estos estudios se han utilizado diferentes métodos de trabajo. Así pues, es difícil discernir si las diferencias son realmente contradictorias. Cadwallader (1979) obtiene una función lineal entre las distancias reales y sus estimaciones empleando metodología diversa.

Los juicios de distancia son generalmente consistentes, como demuestran las relaciones que mantienen con la distancia real, pero no son del todo precisos. Los sujetos cometen errores de infraestimación y sobreestimación, lo que nos indica que la analogía cartográfica no es del todo adecuada. En un plano meramente descriptivo, se ha indicado que la dirección de las estimaciones ejerce influencia sobre los errores, aunque sin precisar la naturaleza exacta de esa influencia. Lee (1970) obtuvo una sobreestimación de la distancia subjetiva hacia las afueras de la ciudad, con respecto a las estimaciones con dirección al centro de la misma. Los experimentos de Golledge et al. (1969) y Briggs $(1973,1976)$ arrojaron resultados opuestos. Los sujetos exageraban las distancias hacia el centro de la ciudad y cometían errores de infraestimación hacia las afueras.

Otra explicación ha considerado el grado relativo de atracción o preferencia de los diferentes lugares, concretamente la valencia afectiva que para el sujeto poseen los lugares de partida y meta (Stea 1969). Los atributos negativos asociados con ciertos lugares inducen a codificarlos más separados de lo que en realidad están (Rapoport 1976). La atracción negativa que ejerce el centro de la ciudad en las ciudades americanas, debido entre otras cosas a la densidad del tráfico, podría explicar los resultados obtenidos por Golledge y Briggs en dichas ciudades. Por el contrario, en los países europeos el centro de la ciudad posee un valor positivo, lo que podría explicar los resultados de Lee. No obstante, otros muchos factores pueden explicar esta relación. Puede ser mucho más fácil realizar estimaciones de distancia en unas ciudades que en otras, debido a su trazado más regular o a la existencia de lugares más distintivos, lo que lleva consigo un incremento de su legibilidad (Canter y Tagg, 1975).

En alguna medida, el grado de familiaridad o conocimiento que se posee de los diferentes lugares puede ser responsable de las distorsiones de la distancia estimada. Se predice una mayor precisión 
a medida que la familiaridad se incrementa. De hecho algunos estudios (Golledge y Zannaras, 1973; Hourihan y Jones 1979) han encontrado que la precisión de los sujetos que habían residido más tiempo en la ciudad era mayor que la de los recién llegados. Es razonable suponer que aquellos individuos que tienen más información acerca del espacio en cuestión sean más exactos en sus estimaciones. Sin embargo, este argumento no es del todo cierto, o al menos esta relación no es completamente exacta. Se precisa un cierta cantidad de familiaridad con el medio para lograr una relativa exactitud, pero la información contextual asociada con los lugares y con la ruta que los conecta introduce distorsiones. La literatura experimental demuestra que existe una relación entre la estructura de la ruta y la estimación de su longitud. La distancia subjetiva se incrementa con la cantidad de lugares intermedios de la ruta (Thorndyke, 1981). El número de giros que existe a lo largo de una ruta influye en la percepción de su distancia. Las rutas que contienen un mayor número de giros se estiman como más largas que otras de igual longitud real, pero con menos giros (Downs y Stea, 1973; Byrne, 1979; Sadalla y Magel 1980). La distancia estimada de la ruta varía también con el número de intersecciones presentes en ella (Sadalla y Staplin, 1980b), así como con la familiaridad de los atributos asociados con éstas (Sadalla, Staplin y Borroughs, 1979). Las rutas que tienen más intersecciones se estiman más largas que otras de distancia objetiva equivalente, pero con un menor número de intersecciones. También se sobreestiman las que poseen nombres más familiares.

La sobreestimación de la distancia por la presencia de lugares intermedios se aprecia también en los juicios de distancia lineal. La distancia subjetiva entre dos objetos localizados en una habitación se incrementa cuando se colocan barreras transparentes u opacas entre ellos (Kosslyn, Pick y Fariello, 1974), aunque los efectos son diferentes con distancias cortas y largas (Sherman et al., 1979; Cohen y Weatheford, 1980). Los resultados indican que tanto niños como adultos cometen este tipo de distorsión en espacios de pequeña escala, como una habitación (Anooshian y Wilson, 1977: Newcombe y Liben, 1982), así como en ambientes de gran escala (Cohen et al., 1978). Se ha comprobado también que la pertenencia categorial introduce distorsión. La distancia entre dos puntos de una figura geométrica se infraestima con respecto a la de otros dos puntos equidistantes, pero incluidos en dos figuras diferentes (Coren y Girgus, 1980).

Por último, la distancia subjetiva viola los supuestos de transitividad y conmutatividad inherentes a la geometría euclidiana. Cadwallader (1979) observó, mediante una tarea de comparación por pares, en la que se requería estimar cuál de dos distancias era mayor, que los sujetos eran incapaces de ordenarlas en un continuo único. Si juzgaban que la distancia «A» era mayor que la distancia «B», 
$y$ « $B »$ mayor que «C», esto no impedía que luego estimasen que «C» era mayor que «A». En otro experimento este mismo autor puso de manifiesto que existían asimetrías en las estimaciones de distancia. La distancia subjetiva entre el lugar «X» y el lugar «Y» no era igual a la que se estimaba entre «Y» $\mathrm{y}$ «X». Un ejemplo cotidiano de este fenómeno lo constituye el que percibamos a veces más corto el trayecto de casa al trabajo que del trabajo a casa o viceversa, aunque sepamos con certeza que la distancia real es la misma. Cadwallader interpreta los resultados en función del grado diferente de atracción que ejercen los dos lugares. Este hallazgo fue corroborado por Sadalla et al. (1980), quienes atribuyen el fenómeno de asimetría a la diferencia de tipicidad o referencialidad geográfica de los lugares (hipótesis de la tipicidad geográfica). La distancia subjetiva desde un lugar poco saliente hasta otro más saliente será menor que a la inversa. Sin embargo, Holyoak y Mah (1982) ofrecen una explicación diferente basándose en la necesidad de una discriminabilidad mayor en las áreas más densas del espacio, cambios de escala inducidos por la naturaleza de los lugares, y en la actuación de los puntos de referencia como mejores indicios en la recuperación de otros lugares (hipótesis de la densidad métrica). Las distancias se sobreestiman cuando empleamos una escala de medida reducida, porque precisamos realizar discriminaciones más finas en las distancias que con una escala más amplia. Así, suponiendo que un lugar prominente evoca en la recuperación lugares más remotos y un mayor número de ellos que otro poco prominente, se sigue que la escala subjetiva se amplía y la distancia se infraestima.

Estas dos interpretaciones del fenómeno de asimetría de las estimaciones de distancia no son de hecho totalmente opuestas. Al igual que otros dos modelos, que abordan el mismo fenómeno en los juicios de similitud semántica, «contraste de atributos» (Tversky, 1977) y «distancia-densidad» (Krumhansl, 1978), basan sus explicaciones en dos parámetros. Uno de estos parámetros, común a ambos modelos e hipótesis, es direccionalidad de los juicios o estimaciones. Los valores del otro parámetro, en un caso la tipicidad o prominencia de los estímulos (hipótesis de la «tipicidad geográfica» y modelo de «contraste de atributos»), en el otro la densidad de los lugares en el espacio o de los puntos en una solución dimensional (hipótesis de la «densidad métrica» y modelo de «distancia-densidad»), pueden de hecho coincidir en aquellas situaciones en que los lugares de referencia o estímulos típicos ocupan las zonas más densas. Así pues, en estos casos tanto de ambas interpretaciones como de los dos modelos se derivan predicciones similares. Sin embargo, no siempre ocurre que los prototipos espaciales estén localizados en las regiones más densas del espacio. Carreiras y De Vega (1984) contrastaron empíricamente las dos explicaciones. Seleccionaron mediante dos estudios normativos lugares típicos y no típicos pertenecientes a dos zonas de la ciudad, una densa y otra no densa. Los 
resultados extraídos de las estimaciones de distancia entre los lugares mostraron una interacción entre los dos factores: tipicidad y densidad. Esto sugiere que existe una relación más compleja que la que postulan las explicaciones, convencionales; de modo que densidad y tipicidad se encuentran implicadas.

La amalgama de resultados expuestos pone de manifiesto que existe una distorsión sistemática en las estimaciones de distancia. Ello implica que es insostenible el supuesto de que una geometría euclidiana subyace a la representación espacial de las relaciones entre todos los lugares. Pero además de refutar este supuesto, los datos sugieren que no sólo almacenamos información estrictamente espacial, sino que otro tipo de información de tipo contextual está presente en el mapa cognitivo. La cantidad de información no-espacial almacenada acerca de una determinada área, como sugiere Milgran (1973), o la diferencia de accesibilidad de esa información con respecto a determinados lugares (Sadalla et al. 1979) podrían ser responsables de las distorsiones encontradas.

\subsubsection{Dirección}

La atención experimental dedicada a las estimaciones angulares o de dirección ha sido menor, aunque últimamente ha cobrado interés. Resulta algo sorprendente el abandono, ya que el concepto de mapa cognitivo fue concebido para describir la representación subyacente a las estimaciones angulares de las ratas (Tolman, 1948). En el estudio de Tolman las ratas elegían la dirección de la senda más corta entre varias posibles, para alcanzar la recompensa, dando muestras de una representación de las relaciones espaciales entre los lugares de partida y meta.

Dos pioneros del campo, Gulliver (1908) y Trowbridge (1913) demostraron que la orientación se rige por un sistema de referencia fijo, y que se presta poca atención a sistemas de referencia abstractos como los puntos cardinales. Trowbridge reconoció además la importancia de la casa como lugar central en el sistema de referencia (domicéntrico), hecho resaltado en investigaciones evolutivas más recientes (Biel, 1982). Algunos estudios aislados han enfatizado el rol de variables diferenciales tales como la edad, familiaridad con la ciudad, cultura (Rapoport, 1977), y la capacidad predictora de los informes introspectivos sobre el sentido de la orientación (Kozlowski y Bryant, 1977).

Un estudio más sistemático de las relaciones de dirección fue emprendido desde la perspectiva evolutiva. (Véase Hart y Moore, 1973; Moore, 1976; Pick y Rieser, 1982; Evans, 1980 para una revisión). Los psicólogos evolutivos han dirigido sus esfuerzos a desarrollar un cuerpo de teoría e investigación sobre los sistemas de referencia, influidos por las directrices piagetianas sobre el desarrollo de la con- 
cepción del espacio en el niño. Piaget distingue tres sistemas de referencia, progresiva y cualitativamente diferenciados. En el período sensoriomotor la orientación con respecto al ambiente es egocéntrica, organizada a partir de la posición del niño y de sus acciones en el espacio. Con la externalización de acciones y el comienzo del espacio representacional surgen sistemas de referencia fijos, centrados en la casa (domicéntrico), y en un pequeño número de rutas no coordinadas, mojones y lugares familiares. No existe una conexión entre la orientación de los diferentes grupos de elementos; cada sistema se construye desde un punto de vista particular. Estas representaciones no coordinadas de partes discretas del espacio comienzan a coordinarse al iniciarse el estadio de las operaciones concretas. Finalmente, emerge un sistema de referencia jerárquicamente integrado y organizado en términos de una estructura geométrica abstracta (espacio euclidiano), que permite coordinar múltiples perspectivas. Esta progresión se aplica no sólo a cambios ontogenéticos, sino también a cambios microgenéticos que ocurren en el aprendizaje de un ambiente nuevo.

Algunos estudios empíricos confirmaron la secuencia de desarrollo de los sistemas de referencia postulada por Piaget (Pufall y Shaw, 1973; Hazen et al., 1978). No obstante, existe controversia acerca de la cronología del cambio de etapas. Se ha demostrado que la edad del cambio depende en gran medida de variables situacionales, tales como la naturaleza de la prueba, la saliencia de los puntos de referencia y la complejidad de la tarea de orientación (Pick y Lockman, 1981). La presencia de puntos de referencia reduce el número de respuestas egocéntricas en niños preoperacionales (Huttenlocher y Presson, 1973; Acredolo, 1976, 1977), y ya a los seis meses puede utilizarse un mojón externo de referencia para la orientación si es extremadamente saliente (Rieser, 1979). Además, los efectos de la práctica y la familiaridad con el lugar donde se realiza la tarea aceleran la utilización de información no egocéntrica (Acredolo, 1978, 1979). Desde una postura más crítica, Spencer y Darwizeh (1981a, 1981b) argumentaron que parte de la dificultad que los niños encuentran para demostrar que no ven el mundo de acuerdo con las categorías del desarrollo establecidas, reside en la complejidad de las instrucciones y el tipo de tareas utilizadas. Abogan por una metodología que no infraestime las capacidades de orientación de los niños y por la necesidad de realizar estudios fuera del laboratorio.

Bajo la perspectiva del procesamiento de la información han proliferado en la última década una serie de estudios que revelan la existencia de errores sistemáticos en los juicios de dirección. Todos coinciden en apuntar que ocurren como consecuencia de un procesamiento normal. Según el principio de economía cognitiva no codificamos la gran cantidad de relaciones espaciales existentes en el mundo externo, sino que nos servimos de una serie de heurísticos 
de codificación que simplifican y esquematizan la información. Esa estructura o esquema mejora el recuerdo, evita una sobrecarga innecesaria de memoria, pero introduce distorsión. Por ejemplo, un sesgo de simplificación, comúnmente apreciado en los dibujos, consiste en suavizar las curvas de los trayectos, asimilándolas casi a una línea recta (Milgran y Joselet, 1976; de Jonge, 1962). Por otra parte, debido a la carencia de conocimiento de relaciones específicas entre todos los lugares, utilizamos estrategias inferenciales en la recuperación. Byrne (1979) encontró que los sujetos familiarizados con su ciudad, dibujaban los cruces de calles con ángulos de 90 grados, mientras que los ángulos reales variaban entre 60 y 70 grados y entre 110 y 120 . Byrne atribuye la distorsión a la presencia de un heurístico de almacenamiento, según el cual codificamos los giros como ángulos rectos. Este mismo sesgo hacia el ángulo recto fue registrado por Chase y Chi (1980) empleando la técnica del dibujo, por Moar y Bower (1983) con el método de triangulación mental, y por De Vega et al. (1983) y Carreiras (1984) en estimaciones directas a escala. Moar y Bower apreciaron también que las estimaciones angulares de tríadas de lugares, aprendidos de un mapa cartográfico y por experiencia con el medio, eran consistentemente no reversibles.

Los datos de Tversky (1981) confirman que éstas y otras distorsiones, como la tendencia a convertir en paralelas calles que no lo son, se producen con trazados geográficos aprendidos en contacto con el medio, con mapas reales y ficticios del mundo y con formas visuales. Tversky justifica este tipo de errores mediante dos heurísticos de codificación, derivados de los principios de organización perceptiva: alineamiento y rotación. Estos dos heurísticos distorsionan las relaciones espaciales al imponer más orden o regularidad de la que realmente existe. El heurístico de rotación afecta a la relación figura-fondo, parte-todo. En la representación tendemos a que los ejes de la figura coincidan con los de la estructura de referencia en que se halla inmersa. Los sujetos del experimento de Tversky recordaban la bahía de San Francisco con orientación Norte-Sur, cuando en realidad es Noroeste-Sureste, o consideraban que el emplazamiento correcto de América del Sur se ajusta a las coordenadas Norte-Sur. Los resultados obtenidos por Loftus (1978) indican que tendemos a sesgar los juicios de dirección hacia ángulos de $0,90,180$ y 270 grados. Pero no sólo la orientación de la estructura de referencia, sino también la de otros lugares influye en la orientación de un lugar determinado. Figuras, objetos y lugares se recuerdan más alineados y organizados entre sí de lo que en realidad están. Por ejemplo, los pares América del Norte-Europa, América del Sur-Africa se perciben más alineados en latitud. Asimismo, se considera que Estados Unidos está localizado exactamente al norte de América del Sur.

Los juicios de relaciones espaciales entre lugares se ven afecta- 
dos también por la estructura supraordinada que los agrupa. Al juzgar las relaciones de dirección entre ciudades, incluidas en unidades geográficas o políticas diferentes, distorsionamos la estimación en función de la relación que mantienen entre sí las unidades geográficas a que pertenecen (Stevens y Coupe, 1978). Estos autores sugieren que tales resultados reflejan la existencia de una representación jerárquica de la información espacial, así como el uso de procesos inferenciales en la recuperación de relaciones que no se han almacenado explícitamente. Otras evidencias posteriores muestran que los tiempos de reacción para verificar afirmaciones acerca de la relación espacial entre dos ciudades es diferente si pertenecen a la misma unidad supraordinada o no (Wilton, 1979; Maki, 1981).

Otros estudios (Evans y Pezdek, 1980; Hintzman et al., 1981) han retomado la aportación de Shepard y colaboradores (Ver Shepard y Cooper, 1982 para una revisión), para investigar la transformación mental de información espacial. Shepard y colaboradores demostraron que el tiempo necesario para verificar si dos objetos bi y tridimensionales eran iguales, es una función lineal monótona creciente del grado de rotación de uno de los objetos. Evans y Pezdek encontraron una relación lineal entre el tiempo de reacción para juzgar si la disposición espacial de una tríada de estados era correcta o falsa, y el grado de rotación de la misma. Los resultados se repetían utilizando como estímulos lugares de un campus, pero solamente cuando los sujetos no lo conocían y estudiaban previamente un mapa del mismo. En el caso de que el aprendizaje se produjese por contacto con el ambiente, ni el tiempo de rotación ni los errores de dirección variaban sistemáticamente con el grado de rotación de las tríadas. El tipo de aprendizaje es responsable, en opinión de los autores, de las diferencias. El aprendizaje experiencial del medio permite la adquisición de una representación que no tiene orientación específica, ya que se aprecia desde múltiples perspectivas. Por el contrario un mapa se memoriza bajo una determinada perspectiva.

Los resultados obtenidos por Hintzman y col. no se ajustan bien a la hipótesis de que la recuperación de la dirección requerida se establezca por rotación mental, independientemente de que el aprendizaje previo sea directo o experiencial. Las estimaciones de 180 grados se muestran inesperadamente rápidas y los errores de inversión fueron frecuentes, fenómeno ya detectado por Hardwick, McIntyre y Pick (1976). Hintzman propone como explicación un modelo dual, combinando mecanismos de búsqueda secuencial al comienzo de la tarea y una estrategia de asociación directa adquirida con la práctica.

La disparidad de resultados de los dos experimentos no sólo reside en los datos. Han utilizado procedimientos diferentes, y lo que es más importante, los estímulos pertenecían en un caso a ambientes de gran escala - estados de una nación y edificios de un cam- 
pus-, mientras que en el otro eran objetos de una habitación. Estas diferencias dificultan el contraste de resultados ya que pueden implicar la presencia de mecanismos cognitivos distintos.

En cuanto al fenómeno de «imágenes en espejo», es decir, los errores de inversión, se ha apreciado que se comportan de forma diferente a la de los errores de abertura angular (De Vega et al., 1983; Carreiras, 1984). La independencia de ambos errores - inversión y abertura-, sugiere la presencia de dos operaciones mentales en la estimación de la dirección entre lugares. Una que indica grosso modo el sentido general de la dirección y otra, más específica que permite calcular la amplitud del ángulo.

\subsection{Información atributivo-contextual}

Desde el trabajo pionero de Lynch, gran parte de la investigación se ha centrado en explorar la composición y organización de los mapas cognitivos. El énfasis ha recaído en la representación de elementos físicos, en una comprensión de la geometría interna del ambiente, pero se ha dedicado poca atención al estudio de aspectos sociales, simbólicos y emocional-evaluativos (Craik, 1973; Herzog, Kaplan y Kaplan, 1982). Este sesgo proviene del mismo Lynch, que definió tres componentes para el análisis de la imagen urbana -identidad, estructura y significado-, pero confinó su análisis a los dos primeros. No obstante, el significado es un elemento crucial para la comprensión del conocimiento ambiental y de la conducta (Harrison y Howard, 1972).

Los procesos mentales, desde la percepción a la planificación, son búsquedas de significado, implicando estructuración y simbolización. La información ambiental es continuamente transformada y utilizada a través de procesos cognitivos y afectivos, en la construcción de una representación interna del ambiente. La representación final no es una representación fiel y exacta del ambiente, sino una aproximación que depende, entre otras cosas, de experiencias anteriores, supuestos, valores y del sistema conceptual. En otras palabras, el ambiente experienciado es significativo. La imagen de la ciudad no sólo refleja la realidad física, sino que constituye una unidad simbólica, que nos proporciona los medios para evocar y manifestar la realidad urbana (Ledrut, 1970; Tuan, 1978; Moore, 1979). Atribuimos significado a los lugares, y éste no está enteramente determinado por las características físicas de los mismos. La información contextual o atributiva, complementaria de la localizacional, nos indica qué tipo de fenómenos ocurren en determinados lugares y les confiere un significado particular. Pero además del aspecto puramente descriptivo o denotativo, abarca un componente emocional-evaluativo o connotativo (Harrison y Sarre, 1971; Downs y Stea, 1973). Así pues, una teoría de la representación ambiental debe co- 
tejar el análisis de la organización espacial interna de ambiente con una comprensión del significado ambiental. Es preciso considerar las relaciones espaciales dentro del conjunto de significados urbanos, ya que concediendo un lugar privilegiado al simbolismo urbano no se enmascaran las relaciones espaciales, sino que se la sitúa.

El significado funcional de los lugares constituye un aspecto esencial de la imaginabilidad del medio. La comprensión y conocimiento de los lugares se facilita cuando existe un significado funcional arraigado, y éste se ve incrementado por la congruencia entre características de la forma física y los atributos de actividades específicas del lugar (Steinitz, 1968). El significado funcional es fundamental debido a la necesidad de conocer lo que acontece y dónde. A medida que el individuo se mueve por la ciudad aprende y elabora un esquema espacial, que refleja algo más que el conocimiento de mojones y sendas. Por ejemplo, adquirimos una topografía mental implícita de estrés, peligrosidad, etc., que nos permite evitar ciertas áreas (Gould y White, 1974). Por otra parte, nuestro campo de actividad se restringe a algunos lugares, el conocimiento que poseemos de otras áreas es vago e impreciso, y a menudo se refiere únicamente a aspectos evaluativos de carácter social, obtenidos a través de otros canales de información. Así pues, incluimos en nuestros esquemas dimensiones simbólicas y significativas. Además, el significado funcional, características físicas y socio-culturales, importancia histórica e indicios visuales, entre otros factores, permiten delimitar una jerarquía de lugares en cuanto a referencialidad, lo que facilita la orientación en el medio (Appleyard, 1969; Sadalla et al., 1980; Evans et al., 1982; Carreiras y De Vega, 1984).

El trabajo realizado acerca de los aspectos semánticos del mapa cognitivo ha sido predominantemente ateórico. Ha habido un énfasis excesivo en la construcción de modelos de preferencia y evaluación, derivados empíricamente mediante técnicas de análisis factorial, análisis de conglomerados, regresión múltiple y escalamiento multidimensional, pero se ha prestado muy poca atención a la teoría.

Dos han sido los acercamientos al estudio del significado ambiental. Uno asume que los aspectos del significado pueden ser capturados a través del lenguaje utilizado para describir lugares. Los estudios realizados bajo este supuesto generalmente han empleado la técnica del diferencial semäntico, debido entre otras cosas a su fácil manejo, para la recogida de datos (Canter, 1969; Lowenthal y Riel, 1972; Burgess y Hollis, 1977). Asimismo, las dimensiones de evaluación, potencia y actividad atribuidas al significado del lenguaje, $\mathrm{u}$ otras análogas como placer, arousal y dominancia (Mehrabian y Russell, 1974) se han utilizado para caracterizar e interpretar el significado ambiental. Sin embargo, la factorialización de los descriptores verbales no siempre se ajusta a esas tres dimensiones (p.e. Canter, 1969), lo que ha puesto en tela de juicio su relevancia conceptual en este campo (Horayangkura, 1978). En opinión de Rus- 
sell y Pratt (1980) y Russell, Ward y Pratt (1981), las dimensiones del diferencial semántico capturan únicamente el componente afectivo del significado ambiental, cuya estructura intentaron aislar y describir; pero otras escalas permiten extraer un componente perceptivo-conceptual presente también en el ambiente. El significado ambiental incluye un componente afectivo y otro perceptivo/cognitivo.

Otro acercamiento intenta captar las dimensiones subyacentes al significado ambiental mediante el empleo de MDS para analizar datos de similitudes entre lugares (Desbarats, 1976; Garling, 1976a, 1976b; Ward, 1977). Se presenta como una alternativa al uso de descriptores verbales, basándose en que al proporcionar a los sujetos una serie de descriptores o calificativos, se corre el riesgo de inducir a respuestas predeterminadas o reducir el espectro del significado ambiental. Así, la utilización de diferentes escalas en distintos estudios podría explicar la no correspondencia de los diversos factores obtenidos.

En un intento de integración de hallazgos anteriores, obtenidos con metodología diversa, Ward y Russell (1981a) llevaron a cabo una serie de experimentos con diferentes métodos de análisis y obtención de datos. Demostraron una notable interrelación entre las dimensiones extraídas mediante seis dominios de respuesta: juicios de similitud, respuestas verbales libres, escalas afectivas, escalas típicas del diferencial semántico, escalas perceptivo-cognitivas y escalas de tendencia conductual. Debido a la interrelación encontrada entre las dimensiones así como entre los atributos, apuntan que el intento de búsqueda de una interpretación para un subgrupo de dimensiones ortogonales es equívoco. Caben diversas interpretaciones razonables de la misma estructura de datos. En su opinión, sería más apropiado caracterizar la representación psicológica del ambiente mediante un espacio geométrico de baja dimensionalidad en el que diversos atributos se representan por vectores oblicuos. Esta estrategia, sin embargo, no impide que persista ambigüedad en la interpretación, por lo que consideran como una alternativa válida al modelo dimensional-geométrico, un modelo de rasgos o características integrados en conjuntos difusos (Tversky, 1977; Mervis y Rosch, 1981). En este caso el ambiente sería representado por paquetes o listas de atributos y la similitud semántica entre lugares vendría determinada por el grado de solapamiento entre rasgos o características que poseen (cfr. Carreiras y De Vega, 1984).

\section{CONSIDERACIONES FINALES}

Desde el trabajo pionero de Tolman (1948) y del impulso que Lynch le ha proporcionado al campo, ha aparecido un considerable número de publicaciones sobre mapas cognitivos. Debido a que los 
investigadores provienen de áreas de conocimiento muy diversas, tales como arquitectura, diseño urbano, geografía, psicología ambiental, evolutiva, cognitiva, etc., se ha producido un fructífero esparcimiento de los tópicos de investigación, que ha enriquecido el área de la cognición ambiental. Sin embargo, la interdisciplinariedad ofrece como contrapartida serios problemas en cuanto a la profundidad teórica. Resulta más difícil la comunicación de aportaciones entre los investigadores, dado que tienden a publicar sus trabajos en las revistas de sus disciplina particular. Así, incluso aun cuando sus contribuciones comparten a veces una estructura conceptual similar, las referencias cruzadas entre áreas son escasas, siendo algunos estudios meras repeticiones de otros. Todo ello redunda en una lentitud de la acumulación progresiva de conocimientos, así como en una debilidad teórica global.

El conocimiento que poseemos del mapa cognitivo es escaso, a pesar de la multitud de datos que sobre él aparecen en la literatura experimental. La ausencia de un marco conceptual para categorizar e interpretar los resultados de la gran cantidad de trabajos realizados es sin duda el otro gran determinante de la experimentación fragmentaria. Bajo esta carencia los juicios sobre el valor, la significación y la relevancia de las investigaciones pierden su sentido, ya que no se posee ese punto de anclaje necesario al que hacer referencia. Además, en alguna medida, se ha perdido la visión inicial de conjunto sobre la naturaleza y función o usos del mapa cognitivo. Por ello, se muestra acuciante el desarrollo de una estructura teórica dentro de la que quepa agrupar y discutir al menos un amplio rango de experimentos, y que permita a su vez encauzar las futuras investigaciones. La concepción del mapa cognitivo como un sistema de conocimiento que engloba información, tanto de carácter localizacional como atributivo-contextual, y que comporta un aspecto ejecutivo, dirigido a la acción conductual constituye, a nuestro entender, el punto de arranque de esa empresa. (cfr. De Vega, 1982).

Hemos observado en las páginas precedentes que una gran parte de las investigaciones pone-el énfasis en la comprensión y el conocimiento de la geometría subjetiva del ambiente, así como en su grado de adecuación con la real. Desde este punto de vista se resalta que el mapa cognitivo proporciona información acerca de la localización relativa de los lugares en el medio, que puede ser utilizada para orientarnos en él. En otras palabras, contiene información sobre el trazado espacial, pertienente para adoptar o decidir sobre estrategias de actuación. Otras, sin embargo, abordan el estudio de la imagen ambiental desde la vertiente de su significado, evaluación y simbolismo, subyaciendo a éstas el supuesto de que imponemos significado al ambiente cuando categorizamos sus elementos. La mayoría de los medios urbanos son demasiado grandes para poder ser apresados desde una sola perspectiva, con lo cual la memoria y el uso de inferencias se muestran necesarios. El usuario cons- 


\section{Estudios}

truye sus esquemas cognitivos, que no solamente enlazan y organizan las percepciones parciales, confiriéndole un sentido de continuidad al espacio, sino que también evalúan y predicen. Así pues, la dicotomía establecida entre las informaciones localizacional y atributivo-contextual constituye dos aspectos de un mismo proceso. La distinción propuesta es válida a efectos de análisis, pero de hecho, es el mapa cognitivo en su globalidad el constructo que nos indica dónde obtener cosas, dónde desarrollar nuestros patrones de actividad, cómo llegar rápido y con facilidad a los lugares, qué sucede en ellos, etc.

Ambos aspectos han sido estudiados por separado, y pocos fueron los intentos de investigar sus interrelaciones de mutua dependencia. Su interacción podría ayudar a explicar, entre otras cosas, la aparición de sesgos e inconsistencias en las estimaciones subjetivas de distancia y dirección, que ha conducido a reavivar la vieja polémica acerca del formato representacional interno (cfr. Carreiras y De Vega, 1984). La información localizacional no se codifica independientemente de la información sobre identidad verbal y visual de los elementos ambientales (Pezdek y Evans, 1979). Es pues preciso concebir el mapa cognitivo como una amplia estructura de referencia en la que una persona organiza actividades, actitudes, conocimiento, que sirve para dar sentido de seguridad emocional, es utilizada para interpretar información nueva y además está dirigida a la acción.

La representación espacial ha sido estudiada por una amplia variedad de razones, siendo la primordial que el ambiente percibido guía nuestra conducta. Sin embargo, paradójicamente, la conexión entre representación y conducta ha recibido muy escasa atención. Ciertamente, una de las funciones clave del mapa cognitivo es la generación de una estructura de referencia o esquema para comprender e interpretar la información procedente del ambiente. Pero, no menos importante es la generación de planes con el fin de resolver problemas espaciales. La información es procesada de forma que pueda ser recuperable y manipulable en cualquier momento para enfrentarnos a un problema como por ejemplo determinar una ruta a seguir, buscar un trayecto alternativo, etc. El mapa cognitivo pierde su valor a no ser que tengamos un proceso para usarlo. Así pues, es preciso establecer un vínculo entre representación y acción, es decir, entre el mapa cognitivo como un sistema de conocimiento espacial y su uso en la coordinación de conductas espaciales adaptativas.

La habilidad para resolver problemas espaciales se basa en la manipulación conjunta de las informaciones localizacional y atributivo-contextual, siendo la clave de tal manipulación la formación y posterior ejecución de un plan. El plan es simplemente una guía, una prescripción de una secuencia de acciones. Tanto la generación o formación del mismo, como su actualización a medida que lo va- 
mos ejecutando en el ambiente presupone la posibilidad de realizar toda una serie de operaciones con la información almacenada en el mapa cognitivo. La formación del plan se controla a través de la comparación de la situación espacial en que se encuentra el sujeto con la representación cognitiva del lugar hacia el que piensa dirigirse, es decir, la meta del plan, cuando ésta no es accesible por percepción directa. Los pasos a dar serían: Identificar, comparando el mapa cognitivo con los indicios perceptivos del lugar en que nos encontramos; recuperar del mapa cognitivo la localización de la meta y los vínculos espaciales entre ésta y el lugar del inicio; finalmente manipular esa información para prescribir una secuencia de instrucciones conductuales.

El proceso completo hasta alcanzar la meta, es decir, la ejecución del plan, encierra una cierta complejidad. El sujeto percibe directamente solo una pequeña parte del ambiente y desde una determinada perspectiva, tanto en la formación del plan, como en los diversos momentos de su ejecución. El espacio es demasiado grande y complejo como para poder apresarlo en su globalidad, es decir, para poder apreciar las relaciones espaciales de localización entre todos los lugares. Además, en la ejecución, las relaciones espaciales entre los diversos lugares implicados y la posición del sujeto cambian continuamente, a medida que éste se desplaza. Así pues, considerando que el sujeto sólo tiene la posibilidad de ver una perspectiva en cada momento y que ésta cambia, para llevar a buen fin el plan formado, es decir, para ejecutarlo y reestructurarlo sobre la marcha si es preciso, debe ser capaz de obtener una visión global del medio, poder rotar el mapa cognitivo y operar sobre él de forma que le permita apreciar el espacio desde una perspectiva múltiple. En suma, la información localizacional debe poder ser transformada para actualizar continuamente la representación de la posición del sujeto con respecto a la de los diversos lugares, especialmente la de la meta, en un ambiente tridimensional que nos rodea. Pero además, no solo basta con transformar y operar de una forma continua sobre las relaciones espaciales, sino que su manipulación debe ser cotejada con las restricciones que impone la información atributivo-contextual incluida en el plan. Cuando generamos un plan para ir a diversos lugares, no siempre cabe la posibilidad de llegar al más lejano habiendo conseguido suplir nuestras demandas primero en los más cercanos, porque restricciones tales como horario de apertura de los lugares, o de otro tipo nos lo impiden. Por ejemplo, supongamos que queremos formar un plan para ir al supermercado, al banco y a correos. Este pudiera ser el ordenamiento óptimo atendiendo únicamente a la variable cercanía espacial desde el punto de partida. Sin embargo, tendremos que reestructurar el plan si correos cierra antes que el banco y el supermercado, y si para comprar en el supermercado tenemos que sacar previamente dinero del banco. 
El proceso de planificación, aunque explorado por algunos estudios (Passini, 1977; Hayes-Roth y Hayes-Roth, 1979), precisa de una mayor atención. Asimismo, otras vías de investigación futura son la optimización o mejora de una serie de condiciones que inciden en y pueden facilitar la formación y uso del mapa cognitivo. Entre ellas cabe citar la legibilidad o articulación perceptiva del medio, la simplicidad de mecanismos auxiliares como el sistema de señalización o los mapas cartográficos, y las estrategias cognitivas de los individuos para recabar información, utilizar los mecanismos auxiliares y planificar.

.La estructura ambiental acota gran parte de la complejidad que puede residir en los mecanismos auxiliares e influye en las estrategias de conocimiento y planificación que adoptan los individuos. Es más fácil la estructuración cognitiva de un ambiente homogéneo. En un ambiente complejo la orientación puede mejorarse con el empleo de un sistema de señalización. Pero para que sea eficiente debe tener en cuenta qué tipo de signos usar, dónde colocarlos y cómo los sujetos pueden aprender a hacer uso de ellos. Por otra parte, los mapas cartográficos son supuestamente una de las herramientas más útiles para ayudar a la gente a orientarse en el medio. Facilitan el establecimiento de una conexión entre el mundo externo y la representación cognitiva. Generalmente emplean convenciones conocidas. Sin embargo, a pesar de su versatilidad a veces nos resulta difícil relacionar los indicios perceptivos del lugar en que nos encontramos con sus correlatos del mapa, o rotarlo para alinearlo con las calles que nos rodean. Es pues preciso ahondar más en la comprensión de los procesos de adquisición, manipulación del mapa cognitivo y planificación, partiendo del hecho de que comprende más que información localizacional y no es una entidad solipsista, sino que tiene un componente ejecutivo.

\section{Resumen}

El articulo propone, desde una perspectiva cognitiva, una estructura conceptual para interpretar la amalgama de resultados obtenidos por la investigación sobre mapas cognitivos. Se describe y discute su carácter interdisciplinar, su semejanza con otros conceptos, los elementos de que consta, el tipo de información que contiene, asi como sus imprecisiones e inconsistencias. Finalmente, se aportan argumentos acerca de la ventaja que supone partir de una concepción del mapa cognitivo como una estructura multirrepresentacional, dirigida a la acción, y se esbozan algunas sugerencias para la investigación futura.

\section{Abstract}

This paper puts forward, from a cognitive outlook, a conceptual framework to integrate some data about the cognitive-mapping process. The interdisciplinary condition of the cognitive map, its elements, contents, bias, inconsistencies and resemblance with other concepts are described and discussed. Finally, arguments are presented about a conception of the cognitive map as a multirepresentational construct pointing towards action, and some topics for further research are suggested. 


\section{Referencias}

ACREDOLO, L.P. (1976): Frames of reference used by children for orientation in unfamiliar spaces. En G.T. Moore and R.G. Golledge (Eds.) Environmental Knowing: Theories Research and Methods. Stroudsburg. Pa: Dowden, Hutchinson and Ross.

- (1977): Developmental change in the ability to coordinate perspectives of a large-Scale space Developmental Psychology. 13, 1-8.

- (1978): Development of spatial orientation in infancy. Developmental Psychology. 14, 224-234

- (1979): Laboratory versus home: The effect of environment on the 9-month-old Infant's choice of spatial reference system. Developmental Psychology. 15, 666-667.

ACREDOLO, L.P.; PICK, H.L. and OLSEN, M.C. (1975): Environmental differentiation and familiarity as determinants of children's memory for spatial location. Developmental Psychology. 11, 495-501.

Allen, G.L.; Kirasic, K.C.; Siegel, A.W. and Herman, J.F. (1979): Developmental issues in cognitive mapping: The selectior and utilization of environmental landmarks. Child Development. 50, 1062-1070.

ANDERSON, J.R. (1978): Arguments concerning representations for mental imagery. Psychological Review. 4, 249-277

APPLEYARD, D. (1969): Why buildings are know: A predictive tool for architects and planners. Environment and Behavior. 1, 131-156.

- (1970): Styles and methods of structuring a city. Environment and Behavior. , 100-118.

ARAGONES, J.I. (1983): Mapas cognitivos de ambientes urbanos: Un estudio empírico sobre Madrid. (Tesis Doctoral). Universidad Complutense de Madrid.

Bartlett, F.C. (1932): Remembering. Cambrigde: Cambrigde University Press.

BIEL, A. (1982): Childrens's spatial representation of their neighbourhood: a step towards a general spatial competence. Journal of Environmental Psychology. 2, 193-200.

Blaut, F. M., Mc Clearly, G. S. and Blaut, A. S. (1970): Euvironmental mapping in young children. Envisonment and Behavior. 2, 335-349.

Bobrow, D.G. and Norman, D.A. (1975): Same principles of memory schemata. En D.G. Bobrow and A.M. Collins (Eds.) Representation and Understanding: Studies in Cognitive Science. N.Y.: Academic Press.

Boulding, K. (1956): The image. Ann Arbor: University of Michigan Press.

BRATFISCH, O. (1969): A further study of the relation between subjetive distance and emotional involvement. Acta Psychologica. 29, 244-255.

BREWER, W.F. and TReyens, J.C. (1981): Role of schemata in memory for places. Cognitive Psycbology. 13, 207-230.

BRIGGS, R. (1973): Urban cognitive distance. En R.N. Downs and D. Stea (Eds.) Image and Environment. Chicago.: Aldine.

- (1976): Methodologies for the measurement of cognitive distance. En G.T. Moore and R.G. Golledge (Eds.). Environmental Knowing: Theories Research and Methods. Stroudsburg. Pa: Dowden, Hutchinson and Ross.

BurgeSS, J.A. and Hollis, G.E. (1977): Personal London. Geographical Magazine. 50, 155-159.

BYRNE, R.W. (1979): Memory for urban geography. Quarterly Journal of Experimental Psychology. 31, 147-154.

CADWAllader, M. (1973): A Methodological examination of cognitive distance. En W.F.E. Preiser (Ed.) Environmental Design Research. (vol. 2) Stroudsburg, Pa: Dowden, Hutchinson and Ross.

- (1976): Cognitive distance in intraurban space. En G.T. Moore and R.G. Golledge (Eds.) Environmental Knowing: Theories Research and Methods. Stroudsburg, $\mathrm{Pa}$ : Dowden, Hutchinson and Ross.

- (1979): Problems in cognitive distance: Implications for cognitive mapping. Environment and Bebavior. 11, 559-576.

CANTER, D. (1969): An intergroup comparison of connotative dimensions in architecture. Environment and Behavior. 1, 37-48.

- (1977): The Psycbology of Place. London: Architectural Press. (trad. cast.) Psicología del lugar. México: Concepto.

CANTER, D. and TAGG, S.K. (1975): Distance estimation in cities. Environment and Behavior. 7, 59-8.

CANTER, D. and CRAIK, K.H. (1981): Environmental Psychology. Journal of Environmental Psychology. 1, 1-12.

CAPEL, H. (1973): Percepción del medio y comportamiento geográfico. Revista de Geografía. 7, 58-148.

CARR, S. and SCHISSLER, D. (1969): The city as a trip. Environment and Behavior. 1, 7-36.

Carreiras, M. (1984): Mapas cognitivos: Interpretación semántica de las distorsiones de distancia y dirección. (Tesis docroral); Universidad de La Laguna.

Carreiras, M. y De Vega, M. (1984): Mapas Cognitivos: Influencia de la tipicidad semántica y la densidad métrica en las asimetrías de distancia. Revista de Investigación Psicológica (en prensa).

CHASE, W.G. and CHI, M.T.H. (1981): Cognitive skill: Implications for spatial skill in large-scale environments. En J.H. Harvey (Ed.) Cognition, Social Behavior, and the Environment. Hillsdale, N.J.: LEA

Clayton, K. and WOODYARD, M. (1981): The acouisition and utilization of spatial knowledge. En J.H. Harvey (Ed.) Cognition, Social Behavior, and the Environment. Hillsdalle, N.J.: LEA

Cohen, R., Baldwin, L.M. and Sherman, R.C. (1978): Cognitive maps of naturalistic setting. Child development. 48, 1704-1707. 
COHEN, R. and WeATHERFORD, D.L. (1980): Effects of route traveled on the distance estimates of children and adults. Journal of Experimental Child Psychology. 29, 403-412.

COREN, S. and GiRGUS, J.S. (1980): Principles of perceptual organization and spatial distortion: The Gestalt Illusions. Journal of Experimental Psychology: Human Performance and Perception. 6, 404-412.

CraiK, K.H. (1973): Environmental Psychology. Annual Review of Psychology. 24, 403-422.

- (1977): Multiple scientific paradigms in Environmental Psychology. International Journal of Psychology. 12, 147-157.

De JONGE, D. (1962): Images of urban areas. Journal of the American Institute of Planners. 28, 266-276.

De VeGA, M. (1979): On interpretative processes in imagery. The Behavioral and Brain Sciences. 2, 551.

- (1982): Los mapas cognitivos, representaciones, planes y parámetros ambientales. Ponencia presentada en Congreso de Barcelona.

De Vega, M.; ARCE, C. y CARReiras, M. (1983): Distorsiones en las distancias y orientaciones en el mapa cognitivo de Santiago de Compostela. Informe interno del Departamento de Psicologia General. Universidad de Santiago.

DESBARATS, J.M. (1976): Semantic structure and perceived environment. Geographical Analysis. 8, 453-467.

DowNS, R.M. (1981): Maps and mapping as metaphors for spatial representation. En G.T. Moore and R.G. Golledge (Eds.) Spatial Representation and Bebavior across the Life Span. N.Y.: Academic Press.

DownS, R.M. and STEA, D. (1973): Cognitive maps and spatial behavior: processes and products. En R.M. Downs and D. Stea (Eds.) Image and Environment. Chicago.: Aldine.

- (1977): Maps in Minds: Reflections on Cognitive Mapping. N.B.: Harper and Row.

EKMAN, G. and BRATFISCH, O. (1965): Subjetive distance and emotional involvement: A psychological mechanism. Acta Psychologica. 24, 430-437.

Evans, G.W. (1980): Enviromental Cognition. Psychological Bulletin. 88, 259-287 (Trad. cast. Estudios de Psicologia, 1983, 14/15, 47-8.

Evans, G.W.; Fellows, J.; ZORN, M. and DoTY, K. (1980): Cognitive mapping and Architecture. Journal of applied Psychology. 65, 474-478.

Evans, G.W. and PezdeK, K. (1980): Cognitive mapping. Knowledge of real-world distance and location information. Journal of Experimental Psychology: Human Learning and Memory 1, 13-24.

Evans, G.W.; SMITH, C. and PEZDEK, K. (1982): Cognitive maps and urban form. APA Journal. Spring, 232-244.

Foss, P.W. (1980): Constructing cognitive maps from sentences. Journal of Experimental Psychology: Human Learning and Memory. 6, 25-38.

GARLING, T. (1976a): A multidimensional scaling and semantic differential technique study of the perception of environmental settings. Scandinavian Journal of Psychology. 17, 323-332.

- (1976b): The structural analysis of environmental perception and cognition: a multidimensional Scaling Approach. Environment and Behavior. 8, 385-415.

GARLING, T; BOOK, A.; LINDGERG, E. and NILSON, T. (1981): Memory for spatial layout of the everyday physical environment: Factors affecting rate of acquisition. Journal of Environmental Psychology. 1, 263-277.

Garling, T.; BooK, A and LINDBERG, E. (1984a) Cognitive mapping of large-scale environments, action plans, orientation and their interrelationships. Environment and Behavior. 1,3-34.

GarlinG, T.; LindBerG, E.; CARREIRAS, M. and BOOK, A. (1984b) Reference systems in cognitive maps. (En preparación).

GARLING, T; BOOK, A; LINDBERG, E. and SAISA, J. (1982): The spatioltemporal sequencing of everyday activities: How people manage to find the shortest path to travel in their home town. Comunicación presentada en la VII International Conference on People and Their Physical Surroundings, Barcelona, Spain.

GARLING, T.; LINDBERG, E. and MANTYLA, T. (1983): Orientation in building: effects of familiarity, visual access, and orientation aids. Journal of Applied Psychology. 68, 177-186.

Gol.DIN, S.E. and THORNDYKE, P.W. (1982): Simulating navigation for spatial knowledges acquisition. Human Factors. 24, 457-471.

GolledGE, R.G. (1978): Learning about environment. En T. Carlstein, D. Parkes and N. Thrift (Eds.) Making Sense of Time. London: Arnold.

Golledge, R.G., BRigGs, R. and Demko, D. (1969): The configuration of distance in intra-urban space. Proceedings of the Association of American Geographers. 1, 60-65.

GoLLEDGE, R.G. and ZANNARAS, G. (1973): Cognitive approaches to the analysis of human spatial behavior. En W.H., Ittelson (Eds.) Environment and Cognition. London: Seminar Press.

GoulD, P. and WhITE, H. (1974): Mental Maps. Baltimore, Md.: Penguin.

GULLIVER, F.P. (1908): Orientation of maps. Bulletin of American Geographical Society, 55-59.

HarDWICK, D.A.; MCINTBRE, C.W. and PICK, H.L. (1976): The content and manipulation of cognitive maps in children and adults. Monographs of the Society for Research in Child Development. 41, 1-55.

HARRISON, J.D. and HOWARD, W.A. (1972): The role of meaning in the urban image Environment and Behavior 4, 389-411.

HARrison, J.D. and SARRe, P. (1971): Personal constrict theory in the measurement of environmental images problems and methods. Environment and Behavior. 3, 351-374.

HART, R.E. and MOORE, G.T. (1973): The development of spatial cognition: A review. En R.M. Downs and D. Stea (Eds.)Image and Environment. Chicago.: Aldine.

HAYES-Roth, B. and HAYES-Roth, F. (1979): A Cognitive model of planning. Cognitive Science. 3, 275-310. 
HAZEN, N.L.; LOCKMAN, J.J. and PICK, H.L. (1978): The development of children's representations of large-scale environments Child development. 49, 623-636.

Hernández, B. (1984): Un estudio descriptivo del mapa de Santa Cruz de Tenerife. Revista de Investigación Psicológica. 1, 193-215.

HeRzOG, T.R.; KAPLAN, S. and KaPLAN, R. (1982): The prediction of preference for unfamiliar urban places. Population and Environment. 5, 43-59.

Hintzman, D.L.; O'DEll, C.S. and ARNDT, D.R. (1981): Orientation in cognitive maps. Cognitive Psychology. $13,149-206$.

HolydaK, K.J. and MAH, W.A. (1982): Cognitive reference points in judgments of symbolic magnitude. Cognitive Psychology. 14, 328-352.

HorAyAnGKura, V. (1978): Semantic dimensional structures: a methodological approach. Environment and Bebavior. 10, 555-584.

HourihaN, J.K. and JONES, L.E. (1979): Distance estimation and cognitive mapping: A multidimensional scaling analysis. The Irish Journal of Psychology. 2, 79-94.

Huttenlocher, J. and Presson, C.C. (1973): Mental rotation and the perspective problem. Cognitive Psychology. 11, 375-394.

Jiménez Burli.lo, F. (1981): Psicología Ambiental. En F. Jiménez Burillo (Ed.). Psicología y medio ambiente. Madrid.: Ceotma.

KAPLAN, S. (1976): Adaptation, structure and knowledge. En G.T. Moore and R.G. Golledge (Eds.) Environmental Knowing: Theories Research and Metbods. Stroudsburg, Pa: Dowden, Hutchinson and Ross.

KEARINS, J.M. (1981): Visual spatial memory in australian aboriginal children of desert regions. Cognitive Psychology. 13, 434-660.

KInTSCH, W. and Dijk, A. (1978): Toward a model of text comprehension and production. Psychological Review. $85,363-394$.

Kosslyn, S.M. (1980): Images and Mind. Cambridge, Mass: Harvard University Press.

Kosslyn, S.M.; PICK, H.L. and Fariello, G.R. (1974): Cognitive maps in children and men. Child Development. 45, 707-716.

KoSSLYN, S.M. and SHWARTZ, S.P. (1977): A simulation of visual imagery. Cognitive Science. 1, $265-295$.

KOZlOWSKI, L.T. and BRYANT, K.J. (1977): Sense of direction, spatial orientation, and cognitive maps. Journal of Experimental Psychology: Human Learning and Memory 4, 590-598.

KrumhansL, C.L. (1978): Concerning the applicability of geometric models to similarity data: The interrelarionship between similarity and spatial density. Psychological Review 85, 445-463.

Kuipers, B. (1978): Modeling spatial knowledge. Cognitive Science. 2, 129-153.

- (1982): The «map in the head» metaphor Environment and Bebavior 14, 202-220.

KULLER, R. (1977a): Psycho-physiological conditions in theatre construction. Comunicación presentada en 8 th World Congress of the International Federation for Theatre Research, Munich.

- (1977b):Social Crowding and the complexity of the built environment - a theoretical and experimental framework. Comunicación presentada en el Symposium Human consequences of Crowding, Antalya, Turquía.

LEDruT, R. (1970): L'Image de la ville. Espaces et Sociétés. 1, 93-106.

LEE, T. (1968): urban neighbourhood as a socio-spatial schema. Human Relations. 21, 241-267.

- (1970): Perceived distance as a function of direction of the city. Environment and Behavior. 2, 40-51.

LIEBLICH, I and ARBIB, M.A. (1982): Multiple representations of space underlying behavior. Bebavior and Brain Sciences 5, 627-659.

LINDBERG, E. (1984): Acquisition of cognitive maps of large-scale environment. (Doctoral Dissertation). University of Umea.

LindBERG, E. and GARLING, T. (1981): Acquisition of locational information about reference points during locomotion with and without a concurrente task: Effects of number of reference points. Scandinavian Journal of Psychology. 23, 107-218.

- (1982): Acquisition of locational information about reference points during locomotion: The Role of central information processing. Scandinavian Journal of Psychology. 23, 107-218.

- (1983): Acquisition of different types of locational information in cognitive maps: Automatic or Effortful processing? Psychological Research. 45, 19-38.

LofTUS, G.R. (1978): Comprehending compass directions. Memory and Cognition. 6, 416-422.

LOWENTHAL, D. and RIEL, M. (1972): The nature of perceived and imagined environments Environment and Bebavior. 4, 189-207.

LOWREY, R.A. (1970): Distance concepts of urban residents. Environment and Bebavior 2. , 52-73.

LUNDBERG, U. (1973): Emotional and geographical phenomena in psycholphysical research. En R.M. Downs and D. Stea (Eds.) Image and Environment. Chicago.: Aldine.

LYNCH, K. (1960) The Image of the City. Cambridge, Mass: MIT Press (trad. cast.) La imagen de la ciudad. Buenos Aires.: Infinito (1974).

MAGANA, J.R. (1978): An empirical and interdisciplinary test of a theory of urban perception. (Doctoral Dissertation). University of California Irvine.

MAK1, R.H. (1981): Categorization and distance effects with spatial linear orders. Journal of Experimental Psychology: Human Learning and Memory. 7, 15-32.

Mehrabian, A. y Russell., J.A. (1974): An approach to Environmental Psychology. Cambridge, Mass.: MIT Press. 
MERvis, C.B. and Rosch, E. (1981): Categorization of natural objects. Annual Review of Psychology. 32, 89-115. Milgram, S. (1973): Capítulo II: Introducción. En W.H. Ittelson (Ed.) Environment and Cognition. N.Y.: Seminar Press.

Milgram, S. and Jodelet, D. (1976): Psychological maps of Paris. En H. Proshansky, W. Ittelson and L. Rivlin (Eds.) Environmental Psychology. N.Y.: Holt, Rinehart and Winston.

Miller, G.A.; Galanter, E. and Pribram, K.H. (1960):Plans and the Structure of Behavior. N.Y., Holt, Rinehart and Winston.

MINSKy, M. (1975): A framework for representing knowledge. En P.H. Winston (Ed.) The Psychology of Computer Vision. N.Y. McGraw-Hill.

MOAR, G.T, and BOWER, G.H. (1983): Inconsistency in spatial knowledge. Journal of the Psychonomic Society. $11,107-113$.

MoorE, G.T. (1976): Theory and research on development of environmental knowing. En G.T. Moore and R.G. Golledge (Eds.) Environmental Knowing: Theories Research and Metbods. Stroudsburg, Pa: Dowden, Hutchinson and Ross.

- (1979): Knowing about environmental knowing: the current state of theory and research on environmental cognition Environment and Behavior 11, 33-70.

NeISSER, U. (1976): Cognition and Reality: Principles and Implications of Cognitive Psychology. San Francisco.: Freeman and Company. (trad. cast.) Procesos cognitivos y realidad. Madrid: Marova (1981) NewCOMBE, N. and LIBEN, L.S. (1982): Barrier effects in the cognitive maps of children and adults. Journal of Experimental Child Psychology. 34, 46-58.

O'KeEFE, J. and NADEl, L. (7978): The Hippocampus as a cognitive map. Oxford: Clarendon Press.

PASSINI, R.E. (1977): Wayfinding: A study of spatial problem solving with implications for physical design. (Doctoral Dissertation). University of Pennsylvania.

Pezdek, K. and Evans, G.W. (1979): Visual and verbal memory for objects and their spatial locations. Journal of Experimental Psychology: Human Learning and Memory 5, 360-373.

PICK, H.L. and LOCKMAN, J.J. (1981): From frames of reference to spatial representations. En L.S. Liben, A.H. Patterson and N. Newcombe (Eds.) Spatial Representation and Bebavior across the Life Span. N.Y.: Academic Press.

PICK, H.L. and RIESER, J.J. (1982): Children's cognitive mapping. En M. Potegal (Ed.) Spatial Abilities: Development and Physiological Foundation. N.Y.: Academic Press, 107-169.

Pufall, P.B. and Shaw, R.E. (1973): Analysis of the development of children's spatial reference systems. Cognitive Psychology. S, 151-175.

PyLySHYN, Z.W. (1981): The imagery debate: Analogue media versus tacit knowledge. Psycbological Review. $88,16-45$.

RAPOPORT, A. (1976): Environmental cognition in cross-cultural perspective. En G.T. Moore and R.G. Golledge (Eds.) Environmental Knowing: Theories Research and Methods. Stroudsburg, Pa.: Dowden, Hutchinson and Ross.

- (1977): Human Aspects of Urban Form. Oxford.: Pergamon Press. (trad. cast.) Aspectos bumanos de la forma urbana. Barcelona.: Gustavo Gili. (1978).

RIESER, J.J. (1979): Spatial orientation of six-month-old infants. Child development. 50, 1078-1087.

RosCH, E. (1975): Cognitive reference points. Cognitive Psychology. 7, 533-547.

RumelHarT, D.E. (1980): Schemata: The building blocks of cognition. En R.J. Spiro, B.C. Bruce and W.F. Brewer (Eds.)Theoretical Issues in Reading Comprebension: Perspectives from Cognitive Psycbology. Linguistics, Artificial Intelligence and Education. Hillsdale, N.J.: LEA.

Rumel.hart, D.E. and Ortony, A. (1977): The representation of Knowledge in Memory. En R.C. Anderson, R.J. Spiro and W.E. Montague (Eds.) Schooling and the Acquisition of Knowledge. Hillsdale, N.J.: LEA.

Russell, J.A. and PRATT, G. (1980): A description of the afective quality atributed to environment. Journal of Personality and Social Psychology, 38, 311-322.

Russell, J.A. and Ward, L.M. (1982) Environmental Psychology. Annual Review of Psychology. 33, $651-688$.

Russell, J.A.; WARD, L.M. and PRATT, G. (1981): Affective quality attributed to environments: a factor analitic study. Environment and Behavior 13, 259-288.

Sadalla, E.K.; BurroughS, W.J. and Staplin, L.J. (1980): Reference points in spatial cognition. Journal of Experimental Psychology: Human Learning and Memory. 6, 516-528.

SADALLA, E.K. and MAGEL, S.G. (1980): The perception of traversed distance. Environment and Bebavior. 12, 65-79

Sadalla, E.K. and STAPLIN, L.J. (1980a): An information storage model for distance cognition. Environment and Bebavior. 12, 183-193.

- (1980b): The perception of traversed distance: Intersections. Environment and Bebavior. 12, 167-182.

SAdalla, E.K.; STAPLiN, L.J. and Burroughs, W.J. (1979): Retrieval processes in distance cognition. Memory and Cognition. 7, 291-296.

Salmaso, P.; BARONI, M.R.; Job, R. and Peron, E.M. (1983): Schematic information, attention, and memory for places. Journal of Experimental Psychology: Learning, Memory and Cognition. 9, 263-268.

Schank, R.C. and ABelson, R. (1977): Scipts, Plans, Goals and Understanding. Hillsdale, N.Y.: LEA

ShemYakin, F.N. (1962): Orientation in space. En B.G. Ananyev et. al. (Eds.) Psychological Science in the URSS. Vol. 1 Part. 1 (Rep. $N^{0}$ 11466). Washington. D.C.: U.s. Office of Technical Reports, 186-255. 
SHePARD, R.N. and COOPER, L.A. (1982): Mental lmages and their Transformations. Cambridge, Mass.: MIT Press.

Sherman, R.C.; Croxton, J. and Glovanatto, J. (1979): Investigating cognitive representation of spatial relationships. Environment and Behavior. 11, 209-226.

Sherman, R.C.; Oliver, C. and TItus, W. (1980): Verifying environmental relationships. Memory and Cognition. $8,555-562$.

SIEGEL, A.W.; KIRASIC, K.C. and KAIL, R.V. (1978): Stalking the elusive cognitive map: The development of children's representation of geographic space. En J.F. Wohlwill and I. Altman (Eds.) Human Behavior and Environment (vol. 3). N.Y., Plenum Press.

Stegel, A.W. and SCHADLER, M. (1977) The development of young children's spatial representations of their classrooms Child development. 48, 388-394.

SIEGEL, A.W. and WHITE, S.H. (1975): The development of spatial representation of large-scale environments. En H.W. Reese (Ed.) Advances in Child Development an Behavior. (vol. 10), N.Y.: Academic Press.

SPENCER, $C$. and DARVIZEH, $Z$. (1981a): The case for developing a cognitive environmental psychology that does not underestimate the abilities of young children Journal of Environmental Psychology 2, 23-35

- (1981b): Young children's descriptions of their local environment: a comparison of information elicited fy recall, recognition and performance techniques of investigation. Environmental Education and information. $1,275-284$.

SPENCER, C. and WeETMAN, M. (1981): The microgenesis of cognitive maps: a longitudinal study of new residents of an urban area. Transactions of the Institute of British Geographers N.S. 6, 375-384.

STEA, D. (1969): The measurement of mental maps: An experimental model for stugying conceptual spaces. En K.R. Cox and R.G. Golledge (eds.) Behavioral Problems in Geography: a Symposium. Evanstons, Nothwestern University Press.

STEINITZ, C. (1968): Meaning and the congruence of urban form and activity. Journal of the American Institute of Planners. 34, 233-247.

Stevens, A. and COUPE, P. (1978): Distortions in judged sparial relations. Cognitive Psychology. 10, 422-437.

STOKOLS, D. (1978): Environmental Psychology. Annual Review of Psychology. 29, 253-95.

THORNDYKE, P.W. (1981): Spatial cognition and reasoning. En J.H. Harvey (Ed.) Cognition, Social Behavior, and the Environment. Hillsdale, N.J.: LEA

THORNDYKE, P.W. and HAYES-ROTH, B. (1982): Differences in spatial knowledge acouired from maps and navigation. Cognitive Psychology. 14, 560-589.

THORNDYKE, P.W. and STASZ, C. (1980): Individual differences in procedures for knowledge acquisition from maps. Cognitive Psychology. 12, 137-175.

Tolman, E.C. (1948): Cognitive maps in rats and men. Psychological Review. 55, 189-208.

TROWBRIDGE, C.C. (1913): On fundamental methods of orientation and imagery maps. Science. 38, 888-897

TuAn, Y. (1978): Sign and metaphor. Annals of Association of American Geographers. 68, 363-372

TVERSKY, A. (1977): Features of similarity. Psychological Review. 84, 327-352.

TVERSKY, B. (1981): Distorsions in memory for maps. Cognitive Psychology. 13, 407-433.

WARD, L.M. (1977): Multidimensional scaling of the molar physical environment. Multivariate Bebavior Research. 12, 23-42.

WARD, L.M. and RuSSELL, J.A. (1981a): The psychological representations of molar physical environments. Journal of Experimental Psychology: General. 110, 121-152.

- (1981b): Cognitive set and the perception of place Environment and Bebavior. 13, 610-632.

WEISMAN, J. (1981): Evaluating architectural legibility: Wayfinding in the built environment. Environment and Bebavior. 13, 189-203.

WILTON, R.N. (1979): Knowledge of spatial relations: The specification of the information used in making inferences. Quarterly Journal of Experimental Psychology. 31, 133-146.

ZaNNARAS, G. (1970): The relation between cognitive structure and urban form. En G.T. Moore and R.G. Golledge (Eds.) Environmental Knowing. Stroudsburg, $\mathrm{Pa} .:$ Dowder, Hutchinson and Ross. 